\title{
LINEAR WELLPOSEDNESS OF THE EVOLUTION EQUATION OF A MIXING STRIP OF TWO FLUIDS WITH INITIALLY SHARP INTERFACE IN A POROUS MEDIUM
}

\author{
BY \\ JEAN DUCHON AND RAOUL ROBERT \\ Institut Fourier C.N.R.S., UMR 5582, 100 rue des Mathématiques, B.P. 74, 38402 \\ Saint-Martin-d'Hères Cedex, France
}

\begin{abstract}
An illposed sharp interface equation, describing the evolution of two well separated fluids in a porous medium, is replaced by a linearly wellposed Cauchy problem for the evolution of a mixing strip described in terms of the level curves of the volume proportion of one fluid.

1. Introduction. Consider two incompressible fluids $F^{ \pm}$moving in a porous medium and satisfying Darcy's law $k \mathbf{v}=-\nabla p+\rho \mathbf{g}$, where $\mathbf{v}(t, x)$ is the velocity field, $p(t, x)$ the pressure, $\rho(t, x)$ the volumic mass $=\rho^{ \pm}$in $F^{ \pm}$, and $k(t, x)=k^{ \pm} \geq 0$ is an effective dynamical viscosity of fluid $F^{ \pm}$. If they are initially separated by a horizontal interface and the heavier fluid is above the other, it is an unstable equilibrium. Assuming a sharp smooth interface for $t>0$ leads to an illposed evolution equation [1]. The simplest way out of this unsatisfactory state of affairs is to assume a mixing strip of size $\propto t$ with an essentially vertical microstructure. In such a "coarse grained" description, the state of the system is given by the volume proportion $s(t . x)$ of. say, the heavy fluid, at time $t$ and space $x$. This model was studied by Otto [2], who proved the stability of the solution corresponding to an initially flat interface [3]. Our aim in this paper is to show that the evolution equation for $s$ is linearly wellposed.
\end{abstract}

2. The smooth interface model. Let us assume that each of the two fluids $F^{ \pm}$ occupies the domain $\Omega_{t}^{ \pm}$and these two domains are separated by a smooth interface $\Gamma_{t}$ :

$$
\mathbf{R}^{d}=\Omega_{t}^{+} \cup \Omega_{t}^{-} \cup \Gamma_{t} \quad(d=2,3) .
$$

To determine the motion of this system of two fluids. we write Darcy's law for each fluid:

$$
k^{ \pm} \mathbf{v}^{ \pm}=-\nabla p^{ \pm}+\rho^{ \pm} \mathbf{g} \quad \text { in } \Omega_{t}^{ \pm}
$$

Received November 15, 2001 and, in revised form, March 18, 2002.

2000 Mathematics Subject Classification. Primary 76S05, 35Q35.

E-mail address: Jean.Duchon@ujf-grenoble.fr

E-mail address: Raoul.Robert@ujf-grenoble.fr 
together with the incompressibility condition $\operatorname{div} \mathbf{v}^{ \pm}=0$, and the conditions at the interface: $\mathbf{v}^{+} \cdot \nu=\mathbf{v}^{-} \cdot \nu$ and $p^{+}=p^{-}$on $\Gamma_{t}$, where $\nu$ denotes a unit normal vector to $\Gamma_{t}$. This of course amounts to writing, in the whole space $\mathbf{R}^{d}$, in the distribution sense: $k \mathbf{v}=-\nabla p+\rho \mathbf{g}$ and $\operatorname{div} \mathbf{v}=0$, where $\mathbf{v}$ is defined as $\mathbf{v}^{ \pm}$on $\Omega_{t}^{ \pm}$and similarly $k(t, \mathbf{x})=k^{ \pm}, p(t, \mathbf{x})=p^{ \pm}$for $\mathbf{x} \in \Omega_{t}^{ \pm}$.

Let us now focus on the case $d=2$; we shall suppose that $\Gamma_{t}$ is the graph of some smooth function $y=u(t, x)$, where from now on $x \in \mathbf{R}$ denotes a coordinate in the horizontal direction, $y \in \mathbf{R}$ is a vertical coordinate increasing upward, and $\Omega_{t}^{-}=\{(x, y)$ : $y<u(t, x)\}$.

To obtain the evolution equation satisfied by $u(t, x)$ we proceed as follows: The kinematic condition $\left(\mathbf{v}^{+}-\mathbf{v}^{-}\right) \cdot \nu=0$ gives

$$
u_{t} / \sqrt{1+u_{x}^{2}}=\mathbf{v}^{-} \cdot \nu
$$

(we chose $\nu$ pointing upward), where $u_{t}, u_{x}$ stand for $\partial u / \partial t, \partial u / \partial x$.

On $\Omega_{t}^{-}$one has $\operatorname{div} \mathbf{v}^{-}=0$ and $\operatorname{curl} \mathbf{v}^{-}=0$, so that $\mathbf{v}^{-}=\nabla \phi^{-}$. Let us denote $\varphi^{-}(t, x)$ as the boundary value $\phi^{-}(t, x, u(t, x))$, so that

$$
\mathbf{v}^{-} \cdot \nu=\nu \cdot \nabla \phi^{-}=\mathcal{F}_{-}(u)\left[\varphi_{x}^{-}\right]
$$

where $\mathcal{F}_{-}(u)$ is an integral operator associated to $u$.

Thus we get $u_{t}=\sqrt{1+u_{x}^{2}} \mathcal{F}_{-}(u)\left[\varphi_{x}^{-}\right]$.

And now we have to express $\varphi_{x}^{-}$in terms of $u$.

For this we exploit the dynamical relation $\operatorname{curl}(k \mathbf{v}-\rho \mathbf{g})=0$, and after a few cumbersome calculations $[4,5]$, we get the integro-differential equation:

$$
u_{t}=\left(\frac{1}{2} H+X\right)\left(\frac{1}{2}+\alpha Y\right)^{-1}\left(-c u_{x}\right),
$$

where $c, \alpha$ are constants:

$$
c=\frac{\rho^{-}-\rho^{+}}{k^{-}+k^{+}} g . \quad \alpha=\frac{k^{-}-k^{+}}{k^{-}+k^{+}},
$$

and $H, X, Y$ are integral operators. $H$ is the classical Hilbert transform

$$
H f(x)=\frac{1}{\pi} \text { p.v. } \int \frac{f(y)}{x-y} \mathrm{~d} y,
$$

and $X$ and $Y$ are integral operators associated to the kernels

$$
\begin{gathered}
X(x, y)=\frac{u(y)-u(x)}{y-x} Y(x, y), \\
Y(x, y)=\frac{1}{2 \pi} \frac{u(y)-u(x)-(y-x) u^{\prime}(x)}{(y-x)^{2}+(u(y)-u(x))^{2}},
\end{gathered}
$$

for $u$ satisfying

$$
\iint\left(\frac{u^{\prime}(x)-u^{\prime}(y)}{x-y}\right)^{2} \mathrm{~d} x \mathrm{~d} y<\infty
$$

the kernels $X$ and $Y$ are square integrable and thus define Hilbert-Schmidt operators on $L^{2}(\mathbf{R})$. Moreover, one can show [4] that for all $\zeta$ such that $|\zeta| \leq 1$, the operator $\frac{1}{2}+\zeta Y$ is invertible. 
Now one can easily linearize Eq. (1) about 0 . We get

$$
u_{t}=-c H u_{x}, \quad u(0, x)=u_{0}(x)
$$

which, using Fourier transform

$$
\hat{u}(t, \xi)=\int e^{-2 \pi i x \xi} u(t, x) \mathrm{d} x
$$

gives

$$
\hat{u}(t, \xi)=e^{-2 \pi c|\xi| t} \hat{u}_{0}(\xi)
$$

We see that for $c \geq 0$ (i.e., $\rho^{-} \geq \rho^{+}$) the Cauchy problem is linearly wellposed, and illposed for $\rho^{-}<\rho^{+}$.

Going on this way one can prove that for $c>0$ the Cauchy problem for Eq. (1) is in fact also nonlinearly wellposed in Hadamard's sense ${ }^{1}$, for $u_{0}^{\prime}$ in the Sobolev space $H^{1}(\mathbf{R})$ with small enough norm (see [5]). This kind of result indicates that Eq. (1) may be considered as a relevant model for the behavior of two fluids initially unmixed if the lighter fluid is above the heavier one, while in the opposite case $(c<0)$, the relevance of (1) as a model of this behavior may, at least, be questioned.

Indeed, one can very well neglect this problem of physical relevance and solve the Cauchy problem with appropriate analytic initial data $u_{0}(x)$, at least locally in time. But it appears that this analytic solution is an unphysical one (in fact, nonentropic in a precise sense) as we shall see now.

3. The two-mixed-phases model. The illposedness of the problem is due to the strong unnatural constraint that the upper heavier fluid and the lower lighter one remain separated by a smooth interface. If this constraint is removed, one possible alternative assumption is that the two fluids can mix intimately. The simplest set of equations modelling the evolution of this system is the following:

At time $t$ and position $\mathbf{x} \in \mathbf{R}^{3}$, we denote $\mathbf{v}^{ \pm}$the velocity of the particles of $F^{ \pm}$, and $s(t, \mathbf{x}) \in[0,1]$ the volume proportion of the fluid $F^{+}$in the mixing. The conservation of $F^{+}$may be written

$$
s_{t}+\operatorname{div}\left(s \mathbf{v}^{+}\right)=0
$$

and that of $F^{-}$

$$
(1-s)_{t}+\operatorname{div}\left((1-s) \mathbf{v}^{-}\right)=0
$$

which together imply the global incompressibility condition

$$
\operatorname{div}\left(s \mathbf{v}^{+}+(1-s) \mathbf{v}^{-}\right)=0 .
$$

We also write Darcy's law for each fluid:

$$
k^{ \pm} \mathbf{v}^{ \pm}=-\nabla p+\rho^{ \pm} \mathbf{g},
$$

where $p$ is the unique pressure of the mixture.

As it is well known, the set of (nonlinear) equations (2), (3) in the unknowns $\mathbf{v}^{+}, \mathbf{v}^{-}, s$, $p$ is of hyperbolic type. Indeed, let us seek solutions which depend only on the "vertical"

\footnotetext{
${ }^{1}$ Wellposedness in Hadamard's sense requires the solution to exist, be unique, and depend continuously on the initial data (the mapping $u_{0} \mapsto u(t)$ should be continuous from $C^{m}$ or $H^{m}$ to $C^{0}$ or $\left.L^{2}\right)$.
} 
coordinate $z\left(\mathbf{x}=x \mathbf{e}_{x}+y \mathbf{e}_{y}+z \mathbf{e}_{z}\right)$ with also "vertical" volocities $\mathbf{v}^{ \pm}=v^{ \pm}(t, z) \mathbf{e}_{z}$, volume proportion $s(t, z)$, and pressure $p(t, z)$. We assume a constant gravity field $\mathbf{g}=-g \mathbf{e}_{z}$, and write $\mathbf{e}_{x} \cdot \mathbf{e}_{y}$. and $\mathbf{e}_{z}$ for mit vectors in the two horizontal and one vertical directions of space. We shall suppose also that the mean velocity $v_{m}=s v^{+}+(1-s) v^{-}$vanishes for $:= \pm x$

In this simplest casc. Ecl. (2) yields

$$
\partial_{z}\left(s v^{+}+(1-s) v^{-}\right)=0
$$

from where

$$
s v^{+}+(1-s) v^{-}=0
$$

and Eq. (3) yiclds

$$
k^{+} v^{+}-k^{-} v^{-}=-\left(\rho^{+}-\rho^{-}\right) g .
$$

Finally one gets the equation

$$
s_{t}+\partial_{z}(f(s))=0,
$$

where $f(s)=\gamma s(1-s) /(1-; \beta s), \uparrow=-\left(\rho^{+}-\rho^{-}\right) g / k^{+}, \beta=\left(k^{+}-k^{-}\right) / k^{+}$.

Now specializing to the (ase $k^{+}=k^{-}$(equal viscosity) and after a change of time scale we can take $\gamma=-1$ (we always suppose $\rho^{+}>\rho^{-}$), so that $f(s)=-s(1-s)$; putting $\sigma=2 s-1$ we finally get the classical Burgers equation:

$$
\sigma_{t}+\partial_{z}\left(\sigma^{2} / 2\right)=0
$$

As it is well known [6]. if we take for initial data a smooth compactly supported function $\sigma_{0}(z)$. a unique regular solution exists during a finite time. But after that time, shocks (i.e.. discontinuities in $\sigma(z)$ ) will appear, so that to get solutions defined for all time $t>0$ one must consider weak solutions (in the distributions sense). The difficulty with weak solutions is that they are eventually nonunique. To recover uniqueness of the solution of the Cauchy problem, one has to add a supplementary condition to distinguish among all weak solutions the correct (or physical) one. It is Lax's entropy condition saying that the characteristic curves must point inward at the shocks. This condition cnsures the uniqueness of a weak solution to the Cauchy problem.

Let us consider the particular case where $\sigma_{0}(z)=1$ for $z>0,=-1$ for $z<0$, that is $s_{0}(z)=1$ for $z>0,=0$ for $z<0$ : the heavier fluid occupies the upper half space $z>0$ at time $t=0$. Then one can easily see that the Burgers equation has two weak solutions:

- the stationary solution $\sigma(t, z)=\sigma_{0}(z)$ for all $t \geq 0$, and

- the (solf similar) solution $\sigma(t, z)=z / t$ for $|z| \leq t,=+1$ for $z>t$, and $=-1$ for $\approx<-t$.

One casily checks that Lax's entropy condition is not satisfied for the first solution, while it is for the second.

Fortunately, Lax's condition allows us to eliminate the first solution which is physically doubtful. However, this unphysical solution is the unique analytic solution of the smooth interface problem (1) corresponding to the Cauchy initial condition $u_{0}=0$. 
4. Evolution equation of a mixing strip. We will consider now the following situation: the two fluids $F^{ \pm}$, which have the same viscosity $k^{+}=k^{-}=k$, are supposed to mix only in a region situated between two smooth surfaces $z=z_{u p}(t, x, y)$ and $z=$ $z_{\text {low }}(t, x, y)$. The heavier fluid $F^{+}$is supposed to occupy the upper part of space.

As a first step we write in a convenient way the equation governing the evolution of the strip.

Let us first notice that the system is entirely known if one knows the function $s(t, x, y, z)$ inside the strip. Indeed, the mean velocity field $\mathbf{v}_{m}=s \mathbf{v}^{+}+(1-s) \mathbf{v}^{-}$is supposed continuous on $\mathbf{R}^{3}$ and is divergence free. Outside of the strip one has curl $\mathbf{v}^{ \pm}=0$ (Darcy's law), and inside

$$
\operatorname{curl} \mathbf{v}_{m}=\nabla s \wedge\left(\mathbf{v}^{+}-\mathbf{v}^{-}\right),
$$

with $\mathbf{v}^{+}-\mathbf{v}^{-}=k^{-1}\left(\rho^{+}-\rho^{-}\right) \mathbf{g}$. Making a change in time scale we may assume $k^{-1}\left(\rho^{+}-\right.$ $\left.\rho^{-}\right)|\mathbf{g}|=1$, so that

$$
\operatorname{curl} \mathbf{v}_{m}=s_{x} \mathbf{e}_{y}-s_{y} \mathbf{e}_{x} .
$$

If we suppose that $s_{x}$ and $s_{y}$ vanish at infinity, the only possible $\mathbf{v}_{m}$ is given by the Biot-Savart formula:

$$
\mathbf{v}_{m}(\mathbf{x})=\frac{1}{4 \pi} \int \frac{\mathbf{x}-\mathbf{x}^{\prime}}{\left|\mathbf{x}-\mathbf{x}^{\prime}\right|^{3}} \wedge\left(s_{y}\left(\mathbf{x}^{\prime}\right) \mathbf{e}_{x}-s_{x}\left(\mathbf{x}^{\prime}\right) \mathbf{e}_{y}\right) \mathrm{d} \mathbf{x}^{\prime} \quad(\mathbf{x}=(x, y, z)) .
$$

Let us now come back to the conservation law in the strip:

$$
s_{t}+\operatorname{div}\left(s \mathbf{v}^{+}\right)=0 .
$$

Let us replace $s \mathbf{v}^{+}$with $s \mathbf{v}_{m}-s(1-s) \mathbf{e}_{z}$ (using the definition of the mean velocity together with $\left.\mathbf{v}^{+}-\mathbf{v}^{-}=-\mathbf{e}_{z}\right)$, which gives

$$
s_{t}+\nabla s \cdot \mathbf{v}_{m}-(1-2 s) s_{z}=0 .
$$

This is a closed equation for $s, \mathbf{v}_{m}$ being given by the kernel (4).

Now it will be convenient to reformulate the problem in terms of the isoconcentration surfaces $z=z(t, \lambda, x, y)$, defined by

$$
s(t, x, y, z(t, \lambda, x, y))=\lambda,
$$

where $\lambda$ varies in the interval $[0,1]$.

Straightforward calculations give the equation satisfied by the function $z$ :

$$
z_{t}+z_{x} v_{1}+z_{y} v_{2}-v_{3}+(1-2 \lambda)=0
$$

where the three components $v_{i}$ of the mean velocity $\mathbf{v}_{m}$ are given by the following:

$$
\begin{aligned}
& v_{1}(\lambda, x, y)=-\frac{1}{4 \pi} \int_{0}^{1} \mathrm{~d} \lambda^{\prime} \int r^{-3}\left[z(\lambda, x, y)-z\left(\lambda^{\prime}, x^{\prime}, y^{\prime}\right)\right] z_{x}\left(\lambda^{\prime}, x^{\prime}, y^{\prime}\right) \mathrm{d} x^{\prime} \mathrm{d} y^{\prime} \\
& v_{2}(\lambda, x, y)=-\frac{1}{4 \pi} \int_{0}^{1} \mathrm{~d} \lambda^{\prime} \int r^{-3}\left[z(\lambda, x, y)-z\left(\lambda^{\prime}, x^{\prime}, y^{\prime}\right)\right] z_{y}\left(\lambda^{\prime}, x^{\prime}, y^{\prime}\right) \mathrm{d} x^{\prime} \mathrm{d} y^{\prime} \\
& v_{3}(\lambda, x, y)=\frac{1}{4 \pi} \int_{0}^{1} \mathrm{~d} \lambda^{\prime} \int r^{-3}\left[\left(x-x^{\prime}\right) z_{x}\left(\lambda^{\prime}, x^{\prime}, y^{\prime}\right)+\left(y-y^{\prime}\right) z_{y}\left(\lambda^{\prime}, x^{\prime}, y^{\prime}\right)\right] \mathrm{d} x^{\prime} \mathrm{d} y^{\prime}
\end{aligned}
$$

where $r=\left[\left(x-x^{\prime}\right)^{2}+\left(y-y^{\prime}\right)^{2}+\left(z(\lambda, x, y)-z\left(\lambda^{\prime}, x^{\prime}, y^{\prime}\right)\right)^{2}\right]^{1 / 2}$. 
We easily recover the mixing strip solution starting with a horizontal interface. Indeed, for $z_{x}=z_{y}=0,(6)$ gives $z_{t}=2 \lambda-1$ so that $z(t, \lambda)=(2 \lambda-1) t$. Notice that now $z \equiv 0$ is no longer a solution of (6), while the corresponding $\sigma=\operatorname{sign}(z)$ was a (nonentropic) weak solution of the Burgers equation.

5. The linearized equation. We consider now the linearization of our problem, about the particular solution $z(t, \lambda)=(2 \lambda-1) t$.

Putting $z(t, \lambda, x, y)=(2 \lambda-1) t+\zeta(t, \lambda, x, y)$, the linearized equation for $\zeta$ (about zero) is easily obtained: $\zeta_{t}=v_{0}$, where

$$
v_{0}(\lambda, x, y)=\frac{1}{4 \pi} \int_{0}^{1} \mathrm{~d} \lambda^{\prime} \int \frac{\left(x-x^{\prime}\right) \zeta_{x}\left(\lambda^{\prime}, x^{\prime}, y^{\prime}\right)+\left(y-y^{\prime}\right) \zeta_{y}\left(\lambda^{\prime}, x^{\prime}, y^{\prime}\right)}{\left[\left(x-x^{\prime}\right)^{2}+\left(y-y^{\prime}\right)^{2}+4\left(\lambda-\lambda^{\prime}\right)^{2} t^{2}\right]^{3 / 2}} \mathrm{~d} x^{\prime} \mathrm{d} y^{\prime} .
$$

We shall show now that Eq. (7) is wellposed in Hadamard's sense.

Let us denote $G(x, y)=-\left(x^{2}+y^{2}+1\right)^{-3 / 2}+3\left(x^{2}+y^{2}+1\right)^{-5 / 2}$; then one readily sees that the vertical velocity perturbation can be written

$$
v_{0}(\lambda, x, y)=\frac{1}{4 \pi} \int_{0}^{1} \mathrm{~d} \lambda^{\prime} \int \frac{1}{\left(2\left|\lambda-\lambda^{\prime}\right| t\right)^{3}} G\left(\frac{x-x^{\prime}}{2\left|\lambda-\lambda^{\prime}\right| t}, \frac{y-y^{\prime}}{2\left|\lambda-\lambda^{\prime}\right| t}\right) \zeta\left(\lambda^{\prime}, x^{\prime}, y^{\prime}\right) \mathrm{d} x^{\prime} \mathrm{d} y^{\prime} .
$$

Now taking the partial Fourier transform of (8) with respect to the variables $(x, y)$ gives

$$
\hat{\zeta}_{t}(t, \xi)=\frac{1}{4 \pi} \int_{0}^{1} \frac{1}{2\left|\lambda-\lambda^{\prime}\right| t} \widehat{G}\left(2\left|\lambda-\lambda^{\prime}\right| t \xi\right) \hat{\zeta}\left(t, \lambda^{\prime}, \xi\right) \mathrm{d} \lambda^{\prime}
$$

for all $\xi$ in $\mathbf{R}^{2}$.

To proceed further we need a technical lemma, the proof of which is postponed until the appendix:

Lemma. We have $\widehat{G}(\xi)=|\xi| \theta(|\xi|)$, where $\theta$ is a $C^{1}$ real function on $[0, \infty)$, satisfying

$$
\begin{gathered}
\forall \delta<1, \exists C_{\delta}>0 \text { such that } \\
|\theta(|\xi|)| \leq C_{\delta} e^{-2 \pi \delta|\xi|} .
\end{gathered}
$$

Now we write our evolution equation for $\hat{\zeta}$ as

$$
\hat{\zeta}_{t}(t, \lambda, \xi)=\frac{|\xi|}{4 \pi} \int_{0}^{1} \theta\left(2\left|\lambda-\lambda^{\prime}\right| t|\xi|\right) \hat{\zeta}\left(t, \lambda^{\prime}, \xi\right) \mathrm{d} \lambda^{\prime} .
$$

For any fixed $\xi,(11)$ is an equation of the form

$$
f_{t}=A(t) f
$$

where $A(t)$ is a bounded linear operator on $L^{2}(0,1)$, with the mapping $t \mapsto A(t)$ continuous for the strong operator topology. It then follows by classical results [7] that this equation has a unique strong solution $f \in C^{1}\left(\mathbf{R} ; L^{2}(0,1)\right)$ satisfying $f(0)=f_{0} \in L^{2}(0,1)$. Henceforth we shall denote $\hat{\zeta}(t, \lambda, \xi)$ the solution of $(11)$ depending on the parameter $\xi$, with initial datum $\hat{\zeta}(0, \lambda, \xi)=\hat{\zeta}_{0}(\xi)$. (Notice that the initial state is a sharp interface between the two fluids; this is why $\zeta_{0}$ does not depend on $\lambda$.)

To see that (11) is wellposed. we will now get some estimates on $\hat{\zeta}$. 
We start from

$$
\hat{\zeta}(t, \lambda, \xi)=\hat{\zeta}_{0}(\xi)+\frac{|\xi|}{4 \pi} \int_{0}^{t} \mathrm{~d} s \int_{0}^{1} \theta\left(2\left|\lambda-\lambda^{\prime}\right||\xi| s\right) \hat{\zeta}\left(s, \lambda^{\prime}, \xi\right) \mathrm{d} \lambda^{\prime}
$$

which gives

$$
|\hat{\zeta}(t, \lambda, \xi)| \leq\left|\hat{\zeta}_{0}(\xi)\right|+\frac{|\xi|}{4 \pi} \int_{0}^{t} \mathrm{~d} s \sup _{\lambda^{\prime}}\left|\hat{\zeta}\left(s, \lambda^{\prime}, \xi\right)\right| \int_{0}^{1}\left|\theta\left(2\left|\lambda-\lambda^{\prime}\right||\xi| s\right)\right| \mathrm{d} \lambda^{\prime} .
$$

Let us denote

$$
C(\alpha)=\sup _{\lambda \in[0,1]} \int_{0}^{1}\left|\theta\left(2\left|\lambda-\lambda^{\prime}\right| \alpha\right)\right| \mathrm{d} \lambda^{\prime}
$$

and

$$
f(t, \xi)=\sup _{\lambda \in[0,1]}|\hat{\zeta}(t, \lambda, \xi)|
$$

One has

$$
f(t, \xi) \leq\left|\hat{\zeta}_{0}(\xi)\right|+\frac{|\xi|}{4 \pi} \int_{0}^{t} f(s, \xi) C(|\xi| s) \mathrm{d} s
$$

from where

$$
f(t, \xi) \leq\left|\hat{\zeta}_{0}(\xi)\right| \exp \left\{\frac{|\xi|}{4 \pi} \int_{0}^{t} C(|\xi| s) \mathrm{d} s\right\} .
$$

Using (10), one easily gets

$$
C(\alpha) \leq C_{\delta} \frac{1-e^{-2 \pi \delta \alpha}}{2 \pi \delta \alpha} \text { for all } \delta<1
$$

so that

$$
\frac{|\xi|}{4 \pi} \int_{0}^{t} C(|\xi| s) \mathrm{d} s \leq \frac{C_{\delta}}{8 \pi^{2} \delta}\left[C_{0}+\log (1+\delta|\xi| t)\right] .
$$

Denote $M=C_{\delta} C_{0} / 8 \pi^{2} \delta$. Using $1+\delta|\xi| t \leq(1+\delta t)(1+|\xi|)$, one gets

$$
|\hat{\zeta}(t, \lambda, \stackrel{\zeta}{\zeta})| \leq M(1+\delta t)^{C_{\delta} / 8 \pi^{2} \delta}(1+|\xi|)^{C_{\delta} / 8 \pi^{2} \delta}\left|\hat{\zeta}_{0}(\xi)\right|
$$

so that for any $\ell>\ell^{*}=\inf _{0<\delta<1} C_{\delta} / 8 \pi^{2} \delta$, one has

$$
|\hat{\zeta}(t, \lambda, \xi)| \leq C(t)(1+|\xi|)^{\ell}\left|\hat{\zeta}_{0}(\xi)\right|
$$

This yields the estimate

$$
\|\zeta(t, \lambda, \cdot)\|_{H^{s-\ell}\left(\mathbf{R}^{2}\right)} \leq C(t)\left\|\zeta_{0}\right\|_{H^{s}\left(\mathbf{R}^{2}\right)}
$$

showing that the linearized Cauchy problem is wellposed on any Sobolev space $H^{s}, s \in \mathbf{R}$.

REMARK. In the case $d=2$, the constants are more explicit in the above proof. One easily gets $\ell^{*}=1 / 2$. 
6. Appendix: Proof of the lemma. The function $G(\mathbf{x})=-\left(\mathbf{x}^{2}+1\right)^{-3 / 2}+3\left(\mathbf{x}^{2}+\right.$ $1)^{-5 / 2}$, defined for $\mathbf{x} \in \mathbf{R}^{2}$, is rotation invariant. Let us write $\widehat{G}(\xi)=4 \pi^{2}|\xi| h(2 \pi|\xi|)$, where $h(\rho)=\rho^{-1} \int_{0}^{x} f(r) \mathcal{I}_{0}(\rho r) \mathrm{d} r$, for $\rho>0 . f(r)=-r\left(r^{2}+1\right)^{-3 / 2}+3 r\left(r^{2}+1\right)^{-5 / 2}$, and

$$
\mathcal{I}_{0}(s)=\frac{1}{2 \pi} \int_{0}^{2 \pi} e^{-i s \cos \theta} d \theta
$$

is the classical Bessel function.

Integrating by parts yields

$$
h(\rho)=\int_{0}^{\infty} \sigma^{2}\left(\sigma^{2}+\rho^{2}\right)^{-3 / 2} \mathcal{I}_{1}(\sigma) \mathrm{d} \sigma,
$$

where we introduce the Bessel function $\mathcal{I}_{1}=-\mathcal{I}_{0}^{\prime}$.

From the above expression for $h$ one can easily deduce that $h(\rho)$ is a $C^{1}$ function on $[0, \infty)$.

Now let us consider the function $F(\mathbf{x})=\left(\mathbf{x}^{2}+1\right)^{-\alpha}, \mathbf{x} \in \mathbf{R}^{2}, \alpha>1$. We derive the following estimate for the Fourier transform of $F$ : for all $\delta, 0<\delta<1$, one has

$$
|\widehat{F}(\xi)| \leq C(\delta) e^{-2 \pi \delta|\xi|},
$$

where $C(\delta)=\pi /(\alpha-1)\left(1-\delta^{2}\right)^{2(\alpha-1)}$.

Indeed, for $\mathbf{z}=\mathbf{x}+i \mathbf{y} \in \mathbf{C}^{2}$, we consider the analytic extension of $F$ in the domain $|\mathbf{y}|<1: F(\mathbf{z})=\left(1+\mathbf{z}^{2}\right)^{-\alpha}$. Using the holomorphy of $F$ we get

$$
\widehat{F}(\xi)=\int_{\mathbf{R}^{2}}\left(1+(\mathbf{x}+i \mathbf{y})^{2}\right)^{-\alpha} e^{-2 \pi i(\mathbf{x}+i \mathbf{y}) \cdot \xi} \mathrm{d} \mathbf{x}, \quad \forall \mathbf{y},|\mathbf{y}|<1 .
$$

For $\xi \neq 0$, we chose $\mathbf{y}=-\delta \xi /|\xi|, \delta<1$, it comes

$$
\widehat{F}(\xi)=e^{-2 \pi \delta|\xi|} \int_{\mathbf{R}^{2}}\left(1+\mathbf{x}^{2}-\delta^{2}-2 i \delta \mathbf{x} \cdot \xi /|\xi|\right)^{-\alpha} e^{-2 \pi i \mathbf{x} \cdot \xi} \mathrm{d} \mathbf{x} .
$$

from where the estimate straightforwardly follows.

We deduce that

$$
|\widehat{G}(\xi)| \leq\left(1-\delta^{2}\right)^{-3 / 2} e^{-2 \pi \delta|\xi|}
$$

Denoting $\theta(\rho)=4 \pi^{2} h(2 \pi \rho)$ and $M=\sup _{0 \leq \rho \leq 1} \theta(\rho)$, we finally get

$$
\theta(|\xi|) \leq C_{\delta} e^{-2 \pi \delta|\xi|},
$$

with $C_{\delta}=\max \left\{\left(1-\delta^{2}\right)^{-3 / 2}, M e^{2 \pi \delta}\right\}$, and the proof is complete.

\section{REFERENCES}

[1] Jean Duchon and Raoul Robert, Estimation d'opérateurs intégraux du type de Cauchy dans les échelles d'Ovsjannikov et application. Ann. Inst. Fourier (Grenoble) 36 (1), 83-95 (1986)

[2] Felix Otto, Evolution of microstructure in unstable porous media flow: A relaxational approach, Comm. Pure Appl. Math. 52 (7) 873-915 (1999)

[3] Felix Otto, Evolution of microstructure: An example, Ergodic theory, analysis, and efficient simulation of dynamical systems, Springer, Berlin, pages 501-522, 2001

[4] Jean Duchon and Raoul Robert. Sur quelques problèmes à frontière libre analytique dans le plan. Séminaire Bony-Sjöstrand-Meyer (Ecole Polytechnique. Palaiseau), 1985

[5] Jean Duchon and Raoul Robert, Perturbation quasi différentielle d'un semi-groupe régularisant dans une échelle d'espaces de Banach, Ann. Inst. H. Poincaré Anal. Non Linéaire 4 (4) 377 - 399 (1987)

[6] Denis Serre, Systèmes de lois de conservation, Diderot éditeur, 1996

[7] Michael Reed and Barry Simon. Methods of modern mathematical physics. I. Functional analysis, Academic Press, 1980 